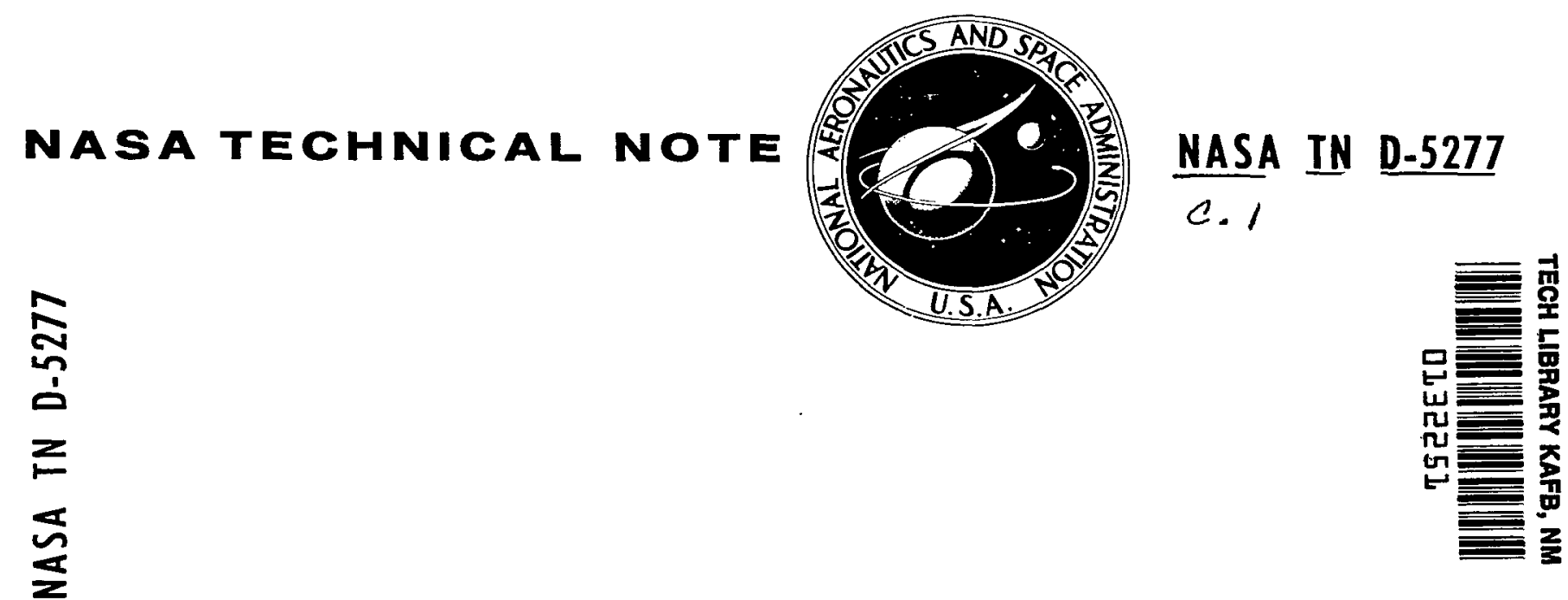

LOAN COPY: RETUKIV AFWL (WLIL-2)

KIRTLAND AFB, N MEX

\title{
A FORMALIZATION OF MEASUREMENT SCALE FORMS
}

by Wayne A. Lea

Electronics Research Center

Cambridge, Mass.

NATIONAL AERONAUTICS AND SPACE ADMINISTRATION • WASHINGTON, D. C. • JUNE 1969 
A FORMALIZATION

$\mathrm{OF}$

MEASUREMENT SCALE FORMS

By Wayne A. Lea

Electronics Research Center

Cambridge, Mass.

NATIONAL AERONAUTICS AND SPACE ADMINISTRATION

For sale by the Clearinghouse for Federal Scientific and Technical Information Springfield, Virginia 22151 - CFSTI price $\$ 3.00$ 


\section{A FORMALIZATION OF MEASUREMENT SCALE FORMS \\ By Wayne A. Lea \\ Electronics Research Center}

\section{SUMMARY}

The general measurement scale hierarchy developed by $\mathrm{S}$. $\mathrm{S}$. Stevens has had widespread use and discussion, but has suffered from various criticisms, many of which may be traced to the informal descriptions and minimal mathematical formulations used in the original definitions and claims. In this paper, a mathematical model of measurement and specific scale forms is developed, using the minimum mathematical structure necessary to define each scale type. Measurement is defined, and a series of restrictions are applied to measurements to yield a precisely specified hierarchy of measurement scale forms including nominal, ordinal, interval, and ratio scales, as well as several others. Equivalences are mathematically defined for each scale form, and theorems are presented specifying the sets of transformations under which equivalence is preserved, thus yielding the scale form invariances discussed by stevens. The hierarchy of measurement scale forms is summarized in a pictorial 'spectrum' diaqram. The development of the model in terms of the structure of possible measurement comparisons (e.g., whether one can establish that one item is 'less' than another, or, alternatively, establish how much the one is less than the other, etc.) is in marked contrast to stevens' definition in terms of scale invariances. This approach avoids several criticisms raised against stevens' approach. One might hope that the alternative approaches would yield corresponding results, and indeed this is found to be generally true. Those cases where differences do occur, however, prove to be vital, and yield several striking unanswered questions about scale forms and their invariances. 


\section{I. - INTRODUCTION}

S. S. Stevens has, in several papers (refs. 1,2,3) relevant to psychophysics, presented a hierarchy of measurement scale forms. These scales have proven of some merit to the general theory of measurement, its implications about appropriate statistical measures, and its application to subjective scaling. Yet, some debate has ensued (refs. 4,5) concerning the validity and preciseness of Stevens' scale forms and associated predictions. One argument (ref. 5) against stevens' hierarchy has centered on the imprecision of the description of the scale forms, their invariances under certain transformations, and the consequent statistical predictions.

In this report, a formalization or mathematical model of Stevens' scales is presented, and some theorems are developed, demonstrating the validity of a number of stevens' results and providing a framework for the derivation of additional theorems. This model has proven of considerable value, for example, in the development of an axiomatic theory of measurement of syntactical complexity of certain formal expressions (ref. 6). Its applicability to other measurement problems should be apparent.

In Section II, the formalization will begin with a definition of measurement which closely relates to the most general definition suggested by stevens in (ref. 1). Postulates restricting the form of measurements are successively applied in sections III to VIII yielding the nominal, ordinal, interval, and ratio scales of measurement as well as several others. Associated definitions and theorems concerning scale equivalences and invariances under sets of transformations (functions) are developed for the various scale types. In section IX, these results are summarized by a pictorial diagram of the hierarchy or 'spectrum' of measurement scale types.

The formalization presented in this report was oriqinally a part of the author's unpublished thesis (ref. 6) and subsequently 
appeared as an unpublished internal NASA memorandum. Reference to that memorandum by S. S. Stevens (ref. 7) led to a volume of requests for the unpublished paper, which in turn has encouraged this publication. Originally the formalization was developed independent of any knowledge of the extensive formalization presented by suppes and zinnes (ref. 8). Subsequent comparisons have disclosed that Suppes and Zinnes have taken a significantly different approach to formalization, and that the arguments in this paper represent an interesting and, it is argued, better alternative model.

The present formalization takes as fundamental the mappings from a domain into a range of measure values and the postulated structure of that range. From such, the notions of scale type, scale equivalences or isomorphisms, and invariances are derived. Major differences from previous models thus include: the definition of scale types in terms of the observed structure of the empirical data or domain, rather than in terms of invariances under certain transformations; the avoidance of numbers as measures until the scale type incorporates most or all of the properties of number systems; and the explicit definition of "equivalences" of two nominal scales, ordinal scales, or whathave-you. In contrast, suppes and zinnes considered as fundamental to their model the concepts of scale type, isomorphisms, and relational systems, and defined scales in terms of invariances. More complete discussion of these differences and their relation to present disputes in measurement theory is forthcoming.

\section{II. - MEASUREMENTS}

The general theory of measurement has provided basic principles for the development of adequate measures of quantities like length, temperature, heat, weight, economic utility, complexity, etc. Within measurement theory (refs. 1-3, 9-16), measurement is specifically defined, its functions discussed, 
and postulates and theorems about acceptable measures are presented. For example, stevens (ref. 1, p. 22) has defined measurement as follows: "Measurement is the assignment of numerals to objects or events according to rules." Thus, measurement is ùsually considered to require the assignment of a number, or a position on a scale, to each of a set of events or items.

Actually, it is conceivable that one could define a measurement process which assigned the members of any set $M$ to the class of items being 'measured.' It need not be that most obvious available class, the real numbers (or any subset of the reals), even though the detailed mathematics available for the set of real numbers justifies its frequent use. In fact, the assignment need not even be in accordance with any explicit rules which assign a fixed set of 'values' for each item being measured. Yet, to have useful properties emerge from the assignment, it is reasonable to require, as stevens does, that the assignment be in accordance with an explicit rule which consistently assigns the same values or measures to each item. Such conditions are, at this point, quite inclusive, so that any assignment which is not random would be allowed as a possible assignment rule.

Thus, it is reasonable to present a general definition that asserts the following description.

Definition 1

A measurement is an (explicit) assignment of a number of elements of a set $M$ to each of the objects or events in a domain D. Thus, a measurement is a binary relation* $R$ such that, for each $I_{j} \varepsilon D, I_{j} R m_{j, i}$ holds for elements $m_{j, i}$ in some subset $M_{j}$ of M. Each element $m_{j, i}$ for which $I_{j} R_{j, i}$ holds will be called a

\footnotetext{
*For description of the properties of binary relations, functions, orderings, and other mathematical terms used in the following discussion, see (ref. 17), Chapter 2, (ref. 18), pp. 5-10, or (ref. I), pp. 4-2I.
} 
measure of $I_{j}$. If $I_{j} R_{j}$ for some $m_{j} \varepsilon M$, we say $I_{j}$ is being measured; the set $D$ is being measured if each $I_{j} \in D$ is being measured.

This definition allows arbitrary "multi-valued assignments" of measures to each item, in accordance with a broad interpretation of stevens' definition. It is obviously quite general, but its generality would appear to be the very cause of its relative uselessness. As shall be seen, providing some further restrictions on the form of measurements will allow the development of an interesting hierarchy of measurement scales and associated theorems.

The class of acceptable assignments will be narrowed by successively adding new postulates which delineate the formal properties of acceptable measures. Thus, important formal properties of measurements will be explicitly presented by the stated postulates and demonstrated theorems. This method has been used to develop a systematic (axiomatic) theory of complexity measurement, by stating theoretical assumptions and theorems which lead to predictions open to empirical verification (ref. 6). In this report, results will apply to any form of measurements satisfying the postulates.

Stevens (ref. 2, p. 26) has pointed out that:

"An operation for determining equality is obviously the first step in measurement, but it is more than that. It is the basis of alz our categorizing and conceptualizing (ref. 19) of alz our coding and recording of information. It...provides the basis of our identifying, recognizing, and labeling ordinary objects. Without this step, no further measurement would be possible."

To be able to provide for any form of equality of measured values, we require that an equality relation be defined on the set $M$ of measures. 
Postulate MP-1. - There is an equality relation = defined on $M$ such that, for any $m_{j}, m_{k}, m_{\ell} \varepsilon M$ :

$$
\begin{aligned}
\text { (i) } & \mathrm{m}_{\mathrm{j}}=\mathrm{m}_{\mathrm{j}} & & \text { (= is reflexive) } \\
\text { (ii) } & \mathrm{m}_{\mathrm{j}}=\mathrm{m}_{\mathrm{k}} \rightarrow \mathrm{m}_{\mathrm{k}}=\mathrm{m}_{\mathrm{j}}^{*} & & \text { (= is symmetric) } \\
\text { (iii) } & \mathrm{m}_{\mathrm{j}}=\mathrm{m}_{\mathrm{k}^{\prime}} \mathrm{m}_{\mathrm{k}}=\mathrm{m}_{\ell} \rightarrow \mathrm{m}_{\mathrm{j}}=\mathrm{m}_{\ell} & & \text { (= is transitive) }
\end{aligned}
$$

Now any practical case of measurement, (e.g., certainly in the case of complexity) would require that the assignment be a single-valued function. That is, for any given item to be measured, such as the length of a rod, a temperature, or the syntactical complexity of a structured expression, a measuring technique which assigned a single value to the item would be desired. Thus, a rod being simultaneously 2 inches long and 43 inches long, a temperature being simultaneously 10 degrees centigrade and 90 degrees centigrade, or a sentence having complexity values of both 2 and 7 of the same units are undesirable. (This, of course, does not exclude the possibility of having separate alternative assignments which say a rod is both 2 feet long and 24 inches long; a temperature is 100 degrees centigrade, 212 degrees Fahrenheit, and 373 degrees Kelvin; or a sentence has a 'depth' of 7 and a 'nesting' of 2 (ref. 6). This use of different units and different additive constants will be discussed below as the possible result of different scales of measurement.)

This reasonable requirement for a single-value measurement can be stated by a general postulate of the form: Postulate MP-2. - Measurements are to be single-valued functions defined on the domain $D$ of measured items and mapping into the chosen set $M$ (in symbols, $f: D \rightarrow M$ ). That is, the assignment is to be a function $f$ which pairs $f\left(I_{j}\right)=m_{j}(\varepsilon M)$ with item $I_{j}$, such that** $f\left(I_{j}\right) \neq f\left(I_{k}\right) \rightarrow I_{j} \neq I_{k}$, for all $I_{j}, I_{k} \varepsilon D$, and $I_{j} \neq I_{k}$ meaning that $I_{j}$ and $I_{k}$ are not the same item of $D$.

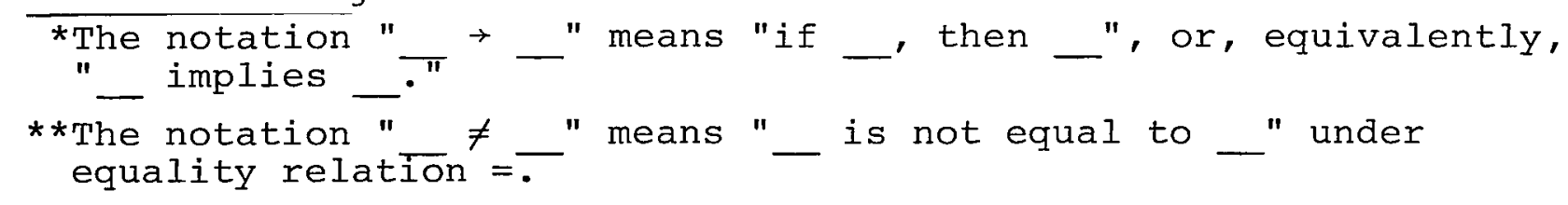


A single-valued function $f$ defines an equivalence relation on the set $D$ of items being measured, so that two items of $D$ are considered 'equivalent' $\left(I_{j} \equiv_{f} I_{k}\right)$ if they have the same measure, or image under the map $f$ :

Definition 2

We will say, for $I_{j}, I_{k} \varepsilon D$, that $I_{j} \equiv_{f} I_{k}$ (read $I_{j}$ and $I_{k}$ are $f$-equivalent," or "I $j$ is f-equivalent to $I_{k}$ ") if, and only if, $f\left(I_{j}\right)=f\left(I_{k}\right)$.

The known properties of the equality relation $(\Leftrightarrow)$ on the set $M$ lead directly to the following theorem. Theorem 1. - The binary relation $\equiv_{f}$ is an equivalence relation. (That is, it is (i) reflexive, (ii) symmetric, and (iii) transitive.)

Proof. Consider $I_{j}, I_{k}, I_{m} \in D$. We need to show:

(i) Reflexivity: $I_{j} \equiv_{f} I_{j}$

Since $f\left(I_{j}\right)=f\left(I_{j}\right)$ by the single-valued property of $f$, then by definition $2, I_{j} \equiv_{f} I_{j}$.

(ii) Symmetry: $I_{j} \equiv_{f} I_{k} \rightarrow I_{k} \equiv_{f} I_{j}$

$I_{j} \equiv_{f} I_{k} \rightarrow f\left(I_{j}\right)=f\left(I_{k}\right)$ by definition 2. Then, by symmetry

of $=$ on $M, f\left(I_{j}\right)=f\left(I_{k}\right) \rightarrow f\left(I_{k}\right)=f\left(I_{j}\right)$. Hence, $f\left(I_{k}\right)=f\left(I_{j}\right)$, and thus, by definition $2, I_{k} \equiv_{f} I_{j}$.

(iii) Transitivity: $I_{j} \equiv_{f} I_{k}, I_{k} \equiv_{f} I_{m} \rightarrow I_{j} \equiv_{f} I_{m}$.

$I_{j} \equiv_{f} I_{k} \rightarrow f\left(I_{j}\right)=f\left(I_{k}\right)$ and $I_{k} \equiv_{f} I_{m} \rightarrow f\left(I_{k}\right)=f\left(I_{m}\right)$ by

definition 2 .

Hence, by transitivity of $=, f\left(I_{j}\right)=f\left(I_{m}\right)$. Thus, by definition 2 again, $I_{j} \equiv_{f} I_{m}$.

Thus, a measurement satisfying Postulate MP-2 defines an equivalence relation $\equiv_{f}$ on $D$. As with any equivalence relation, we can define equivalence classes $\left[\mathrm{I}_{j}\right]=\left\{\mathrm{I}_{k} \mid \mathrm{I}_{j} \bar{\Xi}_{f} \mathrm{I}_{k}\right\}$, and the set $D / \equiv_{f}$ of equivalence classes (D/ $\equiv_{f}=\left\{\left[I_{j}\right] \mid I_{j} \varepsilon D\right\}$ ) (cf. ref. 20, p. 2).

*We shall use the symbol /// to mark the end of a proof. 
Figure la illustrates pictorially the case of a multi-valued assignment such as is excluded by Postulate MP-2, while Figure 13 shows one possible (acceptable) single-valued assignment.

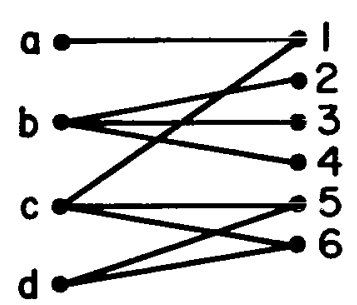

DOMAIN D (a) RANGE M

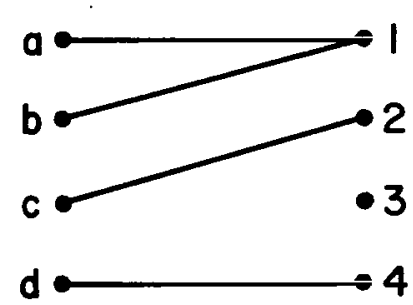

DOMAIN D (b) RANGE M

Figure 1. - Multi-valued and single-valued assignments

Postulate MP-2 prevents the "fanning-out" from the domain D (as shown in Figure la from item $\mathrm{c}$ to 1,5 , and 6 , from b to 2, 3 , and 4, and from d to 5 and 6 ), but does not eliminate the possible fanning into the range, as illustrated (for $a$ and $b$ to value 1 ) in Figure $1 \mathrm{~b}$. The latter would be eliminated by restricting assignments to one-one functions (refs. 1,2,21; p. 176). However, in the sense in which the domain is the set of objects to which properties are attributed and measured (and the domain is not the intuitive class properties which are quantitatively described by the measurements), the measurements are not sensibly assigned by one-one functions. One-one functions would require that only one object could have each value. But, clearly, there are an indefinite number of objects which may have any given value of length, temperature, weight, or complexity.

On the other hand, one could define a one-one function from the set $\mathrm{D} / \equiv_{f}$ of equivalence classes resulting from equivalence relation $\equiv_{f}$ to the set $M$ of measurement values. (This is effectively what Russell (ref. 21, p. 33) suggests and stevens (refs. 1,2) does.) The results of such a map would be similar to those of the map directly from $D$ to $M$, with the exception of the new map making no distinction whatever between members of each class. But, when we consider comparing two or more alternative measurements which give different (intersecting) equivalence 
classes, we will wish to consider the individual items, since two items which are in the same equivalence class under one measurement may not be in the same class under another measurement. Hence, for the purposes of developing a theory capable of comparing alternative measures, it will be useful to consider both the single-valued assignments from $D$ to $M$ and the one-one functions from the set $\mathrm{D} / \equiv_{f}$ of equivalence classes to $\mathrm{M}$.

\section{III. - NOMINAL MEASUREMENT SCALES}

At this point, we have reached substantially the restrictions imposed by "nominal" scales, the broadest (least restrictive) class of measurements (or, measurement 'scales') proposed by Stevens in his extensive work (refs. 1,2) on measurement theory. Thus, based on Stevens' work (ref. 1, pp. 25-26, ref. 2, p. 25), we may define a nominal measurement as follows:

Definition 3

A nominal measurement is one which satisfies Postulates MP-1 and MP-2. A nominal scale is the set D/ $\equiv_{f}$ of equivalence classes set up by a nominal measurement $\boldsymbol{E}$.

Stevens' approach has been to "classify scales of measurement in terms of the group of transformations that leave the scale form invariant" (ref. I, p. 23). For example, if one has a measurement scale or assignment of values which is simply classificatory or nominal, so that no interrelationships between members of the domain $D$ are defined except equivalence of elements, one obtains stevens' "nominal scale". In accordance with Postulate MP-2, the assignment for a nominal scale essentially says merely that one must not assign two or more different numbers to the same member of D. (Also, it follows directly from the definition of $\equiv_{f}$ and the associated equivalence classes that one cannot assign the same measure to two distinct equivalence classes.) Thus, any single-valued function defined on the domain D gives a nominal scale. 
As a step toward formalizing Stevens' concept of scale invariance, we may define what it means for two scales to be nominally equivalent.

Definition 4

Nominal measurements $f$ and $g$ are nominally equivalent if and only if for all $I_{j}, I_{k} \varepsilon D, f\left(I_{j}\right)=f\left(I_{k}\right) \leftrightarrow g\left(I_{j}\right)=g\left(I_{k}\right)$ ** That is, two measurements are nominally equivalent if and only if they partition $D$ into the same equivalence classes: $D / \equiv_{f}=D / \equiv_{g}$. Then, invariance for the nominal scale may be defined:

\section{Definition 5}

A nominal measurement scale $D / \equiv_{f}$ is said to be nominally invariant under a set $T$ of transformations if and only if, for all tET, map $f$ and composite map $t \cdot f$ are nominally equivalent, so that $D / \equiv_{f}=D / \equiv_{t \cdot f}$, where $D / \equiv_{t \cdot f}$ is the set of equivalence classes resulting from the composite measurement $t \cdot f: D \rightarrow M$. (In other words, D/ $\equiv_{f}$ is nominally invariant under $T$ if and only if, for all $t \varepsilon T$ and all $I_{j}, I_{k} \varepsilon D, f\left(I_{j}\right)=f\left(I_{k}\right) \longleftrightarrow$ $\left.t\left[f\left(I_{j}\right)\right]=t\left[f\left(I_{k}\right)\right].\right)$

Stevens' comments (refs. 1,2) to the effect that nominal scales are invariant under the set of one-one functions may now be formalized.

Theorem 2. - A nominal scale is nominally invariant under the set** of all (and only the***) one-one functions defined on the set of images of $f$.

*The expression form $A \leftrightarrow B$, meaning " $A$ if and only if $B$," is equivalent to $\mathrm{A} \rightarrow \mathrm{B}$ and $\mathrm{B} \rightarrow \mathrm{A}$.

**Actually, the set of all such one-one functions form a group (ref. 2, pp. 17-19) under the operation of function composition.

***It should be obvious from definitions 3 and 5 that the set of one-one functions is the largest set of functions which, operating on one nominal scale, yield another nominal scale which is equivalent to the given scale. The condition $f\left(I_{j}\right)=f\left(I_{k}\right) \rightarrow t \cdot f\left(I_{j}\right)=t \cdot f\left(I_{k}\right)$ requires that $t$ be single valued, and the condition $t \cdot f\left(I_{j}\right)=t \cdot f\left(I_{k}\right) \rightarrow f\left(I_{j}\right)=f\left(I_{k}\right)$ requires that $t$ be one-one. 
Proof. - Let $f: D \rightarrow M$ be a nominal measurement, and $g(x)$ be any one-one function defined on the set of images of $f$, so that $g\left[f\left(I_{j}\right)\right]$ is defined for all $I_{j} \varepsilon D$. Define $h: D \rightarrow M$ such that, for $a I I I_{j} \in D, h\left(I_{j}\right)=g\left[f\left(I_{j}\right)\right]$. Then $I_{j} \equiv_{f} I_{k} \rightarrow f\left(I_{j}\right)=f\left(I_{k}\right)$, and thus, by the single-valued property of $g, g\left[f\left(I_{j}\right)\right]=g\left[f\left(I_{k}\right)\right]$. Now, if $I_{j} Z_{f} I_{k}$, then $f\left(I_{j}\right) \neq f\left(I_{k}\right)$ by definition 2 , and hence $g\left[f\left(I_{j}\right)\right] \neq g\left[f\left(I_{k}\right)\right]$ by the one-one property of $g$. According to the paranthetical clause in definition 5, this is sufficient to prove that the scale in invariant under $g$, but we may also go one step more to note that thus $h\left(I_{j}\right) \neq h\left(I_{k}\right)$, and hence $I_{j} \equiv_{f} I_{k} \leftrightarrow I_{j} \equiv_{h} I_{k}$. Since this is true for all $I_{j}, I_{k} \varepsilon D$, we have $D / \equiv_{f}=D / \equiv_{h}$, and hence the scale is indeed invariant under $g$. Since $g$ was an arbitrary one-one function on the image set $f(D)$, the nominal scale is invariant under all such one-one functions. $1 / 1$

Hence, we can say that the nominal scale is of a form such that any single-valued function on D yields a nominal scale (see p. 10), and operating on any given nominal scale with a one-one function will yield another nominal scale of the same form (same equivalence classes.)

A common example of a nominal scale is the numbering of football players on a team. However, in contrast to the practical case of a one-one mapping for players, we would not require that each player have a different number, for an arbitrary nominal scale. We would merely require that any one player have only one number assigned to him. An example which corresponds more closely with reality (i.e., with arbitrary single-valued functions) is the assignment of type or model numbers to classes of items, such as on TV sets, radios, etc. Each class of items has an assigned number, but no class or item is assigned more than one model number.

An important practical example of a nominal measurement scale has been presented (ref. 6, pp. 79-81). The importance 
of this example to electrical engineering and the theory of computation justifies its careful consideration here, since it displays a practical manifestation of the present mathematical model. This example is concerned with the assignment of "state variable" values in switching circuits and associated Boolean expressions.

Boolean expressions representing the behavior of a switching network are formed by recursive processes of Boolean addition and multiplication of state variables (refs. 22,23). The state variables represent the possible "open" or "closed" states of switches, or the "on" or "off" conditions of signals in a gate network. The actual assignment of numerical state values to the switches or gate terminals is an interesting example of nominal scale assignments. As Caldwell (ref. 22, p. 35) explains the matter:

"... a given variable symbolizes the condition or state of a circuit element or a group of circuit elements; it has no numerical significance or value because there is nothing about a condition or state which has universally measurable meaning. We can say that a circuit is turned on or that it is turned off, but we cannot answer the question 'How much is "on" and how much is "off",' except to say that it is alz on or alz off."

All one can say, a priori, is that the two assumed states are distinguishable, like nonequivalent classes or items measured by a nominal scale. Thus, a state variable is representable by a nominal measurement, in which any given position of a switch or value of a voltage (or current) will be assigned one or the other state "value." The two states may be arbitrarily labelled "0" and "1", or "off" and "on", "open" and "closed", etc., as shown in Figure 2. There is nothing sacred about any of these pairs of labels since even a (consistent) reversal of the two labels will not affect the algebra of switching circuits, as long as the labels are likewise reversed in the postulates of the switching algebra (ref. 22, pp. 33-45). 


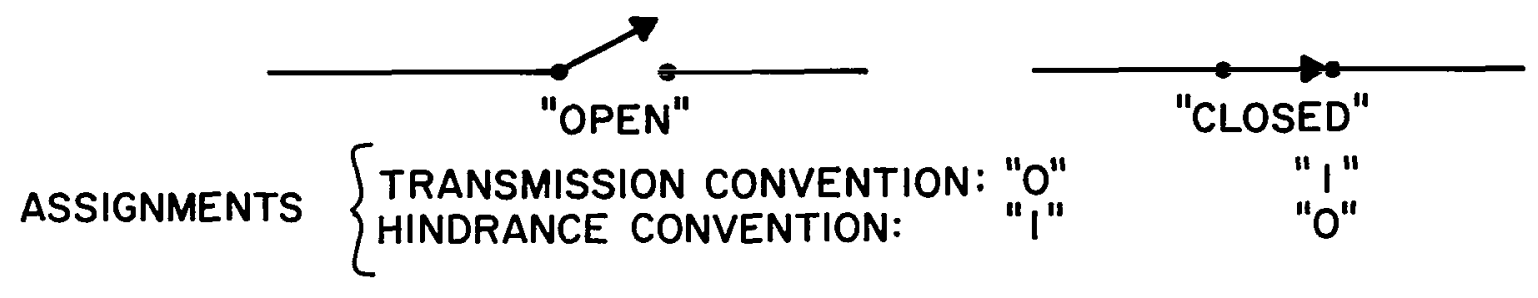

Figure 2. - State variables illustrate nominal measurements

The state labels are selected purely for convenience and to correspond with natural descriptions of physical networks. Yet, even such physical correspondence is not unique, as the use of "transmission" and "hindrance" conventions demonstrates (Figure 2, ref. 22, pp. 45-49 or ref. 23, Sections 2.2 and 2.3).

For gate networks, it is likewise a matter of convention whether a "l" will correspond to a voltage of +Vo, 0 , or -Vo and a "0" will correspond to 0 or $-V o, \pm V o$, or $+V o$, respectively (ref. 23, section 2.4). This again illustrates the nominal equivalence of any such alternative choices or conventions.

In summary, then, it is clear that the state variable assignment in Boolean expressions is a good practical example of a (rather simple) nominal measurement. However, in this example, as well as those of the football players and appliance model numbers (as in most examples given for nominal scales (ref. 1 , p. 25, ref. 2, p. 25)), the assumption is often made that the image set $M$ is the set of real numbers (or a subset of the reals). But this need not be. The numbers assigned as measures are used only as labels or category symbols, and words or letters or any of many other symbols might serve as well. It is only as we consider ordering and other properties exhibited by the real numbers that we see the usefulness of $M$ being considered as the reals or some subset of them. 


\section{IV. - ORDER SCALES}

Stevens recognized the value of measurement scales which involved ordering of the measure values, and from this came his "ordinal scales", which will be discussed in section 5 . These scales involved both ordering and the equivalence relation stipulated by Postulate MP-2. However, it is appropriate here to consider briefly a type of scale which he did not explicitly discuss and which is more general than his ordinal scales. This is the order scale, which satisfies the following postulate:

Postulate MP-2**. - There is a partial ordering relation < defined on $M$, such that, for some (but not all) $m_{j}, m_{k} \varepsilon M, m_{j}<m_{k}$ (i.e., $m_{j}$ "is less than" $m_{k}$ ).

We may now define an order measurement and scale.

Definition 6

An order measurement $\mathrm{f}$ is one satisfying Postulate MP-2' (so that for the set $M$, there exists a partial ordering of measures, but with no requirement that an equivalence relation be defined on the set of measures.) An order scale is the set 0 of sets of elements which are less than each element of $M$ : $0=\left\{A_{j} \mid 1 \leq j \leq n\right\}$ where $A_{j}=\left\{m_{j} \mid m_{i}<m_{j}\right\}$ and $M=\left\{m_{j} \mid 1 \leq j \leq n\right\}$. Equivalence and invariance may now be defined for order measurements and scales.

Definition 7

Order measurements $f$ and $g$ are order equivalent if, and only if, $f\left(I_{j}\right)<f\left(I_{k}\right) \longleftrightarrow g\left(I_{j}\right)<g\left(I_{k}\right)$ ***

*This postulate will be used only in this section, to define and discuss order scales. Consequently, it is given a number MP-2' (rather than MP-3) which is different from the other postulates, so that the number of the other postulates may form a complete consistent sequence.

**A weaker condition has also been shown to be useful in some measurement studies (ref. 6, p. 103). It is: Measurement $g$ is order-consistent with measurement $f$ if, and only if, $f\left(I_{j}\right)<f\left(I_{k}\right) \rightarrow g\left(I_{j}\right)<g\left(I_{k}\right)$. 
An order scale is said to be order invariant under a set $T$ of transformations if, and only if, for all $t \varepsilon T, t$ and $t \cdot f$ are order equivalent (i.e., $f\left(I_{j}\right)<f\left(I_{k}\right) \longleftrightarrow t \cdot f\left(I_{j}\right)<t \cdot f\left(I_{k}\right)$ ). Thus, under order equivalence and invariance, only the relative ordering of items is important, and nothing is said about equivalence of elements under the two measurements*. This is in marked contrast to the ordinal scales of stevens, which will now be considered.

$$
\text { V. - ORDINAL SCALES }
$$

Order scales neglect any equivalences between elements of the measured set. And, on the other hand, the nominal scale does not allow us to say much of quantitative interest. For example, the definition of a nominal scale does not provide for any relative ordexing of the elements. Hence, for such a scale, one cannot meaningfully say "item $I_{j} i s$ longer than Item $I_{k}$ ", "I $I_{j}$ is more complex than $I_{k}$ ", etc.

Actually, to achieve the interesting case in which items of the domain are ordered, we need to add the necessary conditions to obtain stevens' ordinal scale. For an ordinal scale, it is possible to determine which of two measured items is greater than the other (in terms of the property being measured), or if they are equivalent. That is, the function $f$ which measures elements of $\mathrm{D}$ defines a complete ordering of the items of $\mathrm{D}$.

In almost all measurements (e.g., in particular, in complexity measurement) (ref. 6), we desire this complete ordering. We do want to be able to say whether an expression $I_{j}$ is more complex than $I_{k}$, or of equal complexity, and we want conditions like transitivity to hold, so if any expression $I_{j}$ is less

\footnotetext{
*This resembles coombs' work with metric scales (refs. $12,13,14$ ) (also, ref. 2, pp. 34-36), for which only ordering of items is defined.
} 
complex than another $I_{k}$, and $I_{k}$ is less complex than $I_{m}$, then $I_{j}$ is less complex than $I_{m}$. Thus, it is reasonable to propose the following postulate.

Postulate MP-3. - There is a total-ordering relation < defined on $M$ such that, for any $m_{j}, m_{k}, m_{\ell} \varepsilon M^{*}$ :
(i) $m_{j} \not m_{j}$
(< is irreflexive)
(ii) $\mathrm{m}_{\mathrm{j}}<\mathrm{m}_{\mathrm{k}} \rightarrow \mathrm{m}_{\mathrm{k}} \not \mathrm{m}_{\mathrm{j}}$
(< is asymmetrical)
(iii) $m_{j}<m_{k}, m_{k}<m_{\ell} \rightarrow m_{j}<m_{\ell}$
(< is transitive)
(iv) $m_{j}<m_{k}$, or $m_{k}<m_{j}$, or $m_{j}=m_{k}$
(< is a total ordering)

The total ordering < of $M$ and the measurement function $f$ :

$D \rightarrow M$ define a natural ordering relation on the set $D$ being measured, as follows.

Definition 9

A binary relation $<_{f}$ is defined on the domain $D$, such that, for nominal measurement $f$ and any $I_{j}, I_{k} \varepsilon D$,

$$
I_{j}<I_{k} \leftrightarrow f\left(I_{j}\right)<f\left(I_{k}\right)
$$

where (the unsubscripted < is the total ordering relation on $M$ postualted in MP-3.

Theorem 3. - The binary $<_{f}$ of Definition 9 is a "quasi-serial"** ordering relation on $D_{i}$ that is, for any $I_{j}, I_{k}, I_{m} \varepsilon D$ :
(i) $I_{j} \not_{f} I_{j}$
(ii) $I_{j}<I_{k} \rightarrow I_{k} \not_{f} I_{j}$
( $<_{f}$ is irreflexive)
(iii) $I_{j}<f I_{k}, I_{k}<f I_{m} \rightarrow I_{j}<f I_{m}$
( $<_{f}$ is asymmetrical)
(iv) either $I_{j}<_{f} I_{k}, I_{k}<I_{j} I_{j}$ or
$\left(<_{f}\right.$ is transitive)
$<$ is a quasi-serial
ofdering)
*The notation "_ $\$$ " means " is not less than ." In considering the irreflexive ('Tess than') order re $\overline{l a t i o n s}$ here rather than the reflexive order relations ('less than or equal to'), we are following the common practice in measurement theory (refs. 1,2,16). Although the chosen irreflexive relations have a somewhat closer relation to empirical operations, either form of relation is adequate.

**A quasi-serial ordering is here defined in (iv) in accordance with Caw's definition (ref. 11). 
Proof. - The proofs of (i), (ii), and (iii) follow directly from Definition 9 and the irreflexive, asymmetric, and transitive properties of <, and parallel the proof of Theorem 1 . To see that either $I_{j}<_{f} I_{k}, I_{k}<_{f} I_{j}$, or $I_{j} \equiv_{f} I_{k}$, consider each contrary case. First, suppose $I_{j} \Varangle_{f} I_{k} \cdot$ Then, by Definition 9, $f\left(I_{j}\right) \notin f\left(I_{k}\right)$. But, by Postulate MP-3 (iv), then either $f\left(I_{j}\right)=f\left(I_{k}\right)$ or $f\left(I_{k}\right)<f\left(I_{j}\right)$; hence either $I_{j} \equiv_{f} I_{k}$ by Definition 2, or else $I_{k}<_{f} I_{f}$ by Definition 9. Similar reasoning shows that $I_{k} \not_{f} I_{j}$ implies that either $I_{k} \equiv_{f} I_{j}$ or $I_{j}<_{f} I_{k}$. Finally, if $I_{j} \exists_{f} I_{k}$, then $f\left(I_{j}\right) \neq f\left(I_{k}\right)$ by Definition 2. Then, by Postulate MP-3 (iv), either $f\left(I_{j}\right)<f\left(I_{k}\right)$ or $f\left(I_{k}\right)<f\left(I_{j}\right)$, and, hence, by Definition 9 , either $I_{j}<_{f} I_{k}$ or $I_{k}<_{f} I_{j} \cdot / / /$ We may now define an ordinal measurement.

Definition 10

An ordinal measurement $f$ is one satisfying Postulates MP-1, $M P-2$, and $M P-3$, so that the measured set is ordered by $<_{f}$ (of Definition 9). An ordinal scale is the totally ordered set D/ $\equiv_{f}$ of equivalence classes. Or, more precisely, the ordinal scale is the pair $\mathrm{D} / \bar{E}_{\mathrm{f}}{ }{ }^{\prime} \mathrm{f}^{\prime} \equiv_{\mathrm{f}}$ consisting of set $\mathrm{D} / \equiv_{\mathrm{f}}$ of equivalence classes and the total ordering relation ${ }_{f}{ }^{\prime} \equiv_{f}$ defined on $D / \equiv_{f}$ such that $\left[I_{j}\right]<{ }_{f}^{\prime} \equiv_{f}\left[I_{k}\right]$ if, and only if, $I_{j}<_{f} I_{k}$. Clearly, each ordinal measurement is a nominal measurement. Measurement is often considered to require at least the properties of the ordinal scale. For example, Caws (ref. 11, p.5) defined measurement as follows:

"Measurement is the assignment of particular mathematical characteristics to conceptual entities in such a way as to permit (I) an unambiguous mathematical description of every situation involving the entity and (2) the arrangement of alz occurrences of it in a quasi-serial order."

A "quasi-serial ordering" is "an order which determines, for any two occurrences, either that they are equivalent with respect to the property in question of that one is greater than the other" 
(ref. 11). Here, Caws' property (1) suggests the single-valued function assignment, and (2) suggests the ordering of an ordinal measurement.

Now, in analogous manner to the establishment of the range of structural invariance for a nominal scale, we may define an equivalence for ordinal measurements.

Definition 11

Ordinal measurements $f$ and $g$ are ordinally equivalent if, and only if, for all $I_{j}, I_{k} \varepsilon D, f\left(I_{j}\right)=f\left(I_{k}\right) \longleftrightarrow g\left(I_{j}\right)=g\left(I_{k}\right)$, and $f\left(I_{j}\right)<f\left(I_{k}\right) \longleftrightarrow g\left(I_{j}\right)<g\left(I_{k}\right)$. That is, two ordinal measurements are ordinally equivalent if, and only if, they are nominally equivalent and establish the same relative ordering of the domain $D$.

Invariance for the ordinal scale may then be defined.

Definition 12

An ordinal scale is said to be ordinally invariant under a set $T$ of transformations if, and only if, for all $t \varepsilon T, f$ and $t \cdot f$ are ordinally equivalent. (Thus, $D / \equiv_{f}$ is ordinally invariant under $T$ if, and only if, for all $t \in T$ and all $I_{j} I_{k} \varepsilon D$,

$$
f\left(I_{j}\right)=f\left(I_{k}\right) \longleftrightarrow t\left[f\left(I_{j}\right)\right]=t\left[f\left(I_{k}\right)\right]
$$

and

$$
\left.f\left(I_{j}\right)<f\left(I_{k}\right) \longleftrightarrow t\left[f\left(I_{j}\right)\right]<t\left[f\left(I_{k}\right)\right] .\right)
$$

Hence, if a set of transformations preserves the equivalences and ordering of a measurement, the ordinal scale is invariant under them. Now, in accordance with stevens' informal comments about the range of invariance of ordinal scales, we may state: Theorem 4. - An ordinal scale is ordinally invariant under the set (and only the set) of all monotonically increasing functions (that is, functions $p$ for which $x<y \rightarrow p(x)<p(y))$. 
Proof. - Let $\mathrm{f}: \mathrm{D} \rightarrow \mathrm{M}$ be an ordinal measurement and $\mathrm{p}$ be any monotonically increasing function (that is, $x<y \rightarrow p(x)<p(y)$ ) defined on the set $f(D)$ of images of $f$, so that $p\left[f\left(I_{j}\right)\right]$ is defined for all $I_{j} \in D$. Since $p$ is a function, it is immediate that $f\left(I_{j}\right)=f\left(I_{k}\right) \rightarrow p\left[f\left(I_{j}\right)\right]=p\left[f\left(I_{k}\right)\right]$. Now, the monotonic function $\mathrm{p}$ must be a one-one function (since, if $\mathrm{x} \neq \mathrm{y}$ and $\mathrm{p}(\mathrm{x})=\mathrm{p}(\mathrm{y})$, then either $\mathrm{x}<\mathrm{y}$ and $\mathrm{p}(\mathrm{x}) \not \mathrm{p}(\mathrm{y})$ or else $\mathrm{y}<\mathrm{x}$ and $\mathrm{p}(\mathrm{y}) \not \mathrm{p}(\mathrm{x})$, in contradiction to the assumed monotone behavior). Thus, by Theorem 2 and Definition 5 ,

$$
f\left(I_{j}\right)=f\left(I_{k}\right) \longleftrightarrow p\left[f\left(I_{j}\right)\right]=p\left[f\left(I_{k}\right)\right]
$$

Now, $f\left(I_{j}\right)<f\left(I_{k}\right) \rightarrow p\left[f\left(I_{j}\right)\right]<p\left[f\left(I_{k}\right)\right]$, by the monotone property of $\mathrm{p}$. Conversely, $\mathrm{p}\left[\mathrm{f}\left(\mathrm{I}_{j}\right)\right]<\mathrm{p}\left[\mathrm{f}\left(\mathrm{I}_{\mathrm{k}}\right)\right] \rightarrow \mathrm{f}\left(\mathrm{I}_{j}\right)<f\left(I_{k}\right)$ by the one-one property of $\mathrm{p}$. Hence,

$$
f\left(I_{j}\right)<f\left(I_{k}\right) \longleftrightarrow p\left[f\left(I_{j}\right)\right]<p\left[f\left(I_{k}\right)\right]
$$

Since this is true for $a_{l l} I_{j}, I_{k} \in D$, and $p$ is an arbitrary monotonically increasing function, the scale is invariant under the set of all monotonically increasing functions defined on $f(D)$.

To see that the set of monotonically increasing functions is the largest set of transformations yielding ordinal equivalence, note that nominal equivalence requires $t$ to be a one-one function (see Theorem 2 and footnote). The added condition $f\left(I_{j}\right)<f\left(I_{k}\right) \rightarrow t\left[f\left(I_{j}\right)\right]<t\left[f\left(I_{k}\right)\right]$ requires $t$ to be monotonically increasing, by definition.

Thus, any monotonically increasing function operating on an ordinal scale gives another ordinal scale with the same equivalence classes and with the relative ordering of items preserved. There is a meaningful sense in which such scales are "equivalent," since if one such scale says two items are equal, so does the other, and if one says item $I_{j}$ is 'less' (less length, lower temperature, less complexity, etc.) than another item $\mathrm{I}_{\mathrm{k}}$, then 
the other scale likewise says $I_{j}$ is less than $I_{k}$. This development of ordinal scales is thus a formalization of the intuitive sense in which two scales are similar if they order the items of the domain in the same relative way.

For a simple example, consider the ordinal scales $f(x)$ and $g(x)$ in Figure 3. Although the two scales assign different values to some of.the items, and although the intervals between the ordered items are not constant, the two scales do maintain the same relative ordering of the items and are thus in this sense equivalent.

\begin{tabular}{|c|r|r|}
\hline$x$ & $f(x)$ & $g(x)$ \\
\hline$a$ & 1 & 1 \\
$b$ & 2 & 3 \\
$c$ & 3 & 43 \\
$d$ & 4 & 91 \\
$e$ & 5 & 192 \\
\hline
\end{tabular}

Figure 3. - Two ordinally-equivalent scales

We can relate ordinal equivalence to nominal and order equivalence by the obvious theorem:

Theorem 5. - Two measurements $f$ and $g$ are ordinally equivalent if, and only if, they are nominally equivalent and order equivalent.

Proof. - The proof follows directly from Definitions 4, 7, and 11 .

\section{VI. - GENERALIZED INTERVAL SCALES}

Although measurements $f$ and $g$ in Figure 3 are ordinally equivalent, there is also an obvious sense in which they differ. In particular, the intervals between measures as assigned by the two scales are not simply related. For the illustrated real number assignments, the interval between $a$ and $b$ is equal to that 
between $d$ and $e$ for measurement $f$, but they are not assigned equal intervals by measurement $g$. In general, ordinal scales may not include any provision for establishing equality of intervals. The operation of equating intervals between measures is the basis of the interval scale. That is, the interval scale is obtained by postulating a stronger relationship between members of $M$ than just a total ordering. Not only is it assumed that one is able to determine if one element is less than another, but also whether or not the interval between two elements $\mathrm{m}_{j}$ and $\mathrm{m}_{\mathrm{k}}$ is equal to that between $\mathrm{m}_{\mathrm{p}}$ and $\mathrm{m}_{\mathrm{q}}$.

Actually, the invariance of the ordinal scale under monotonic transformations (Theorem 4) suggests the possibility of preserving ordinal scale form under the very simple case of scale translations, or uniform shifting of values. This is, in fact, the very process involved in empirically comparing intervals between measures. When one, for example, wants to see if rod (a) is as much longer than rod (b) as rod (c) is than rod (d); one can mark the intervals on the long rods, place the intervals side by side, and attempt to match both ends simultaneously (just as he does in comparing any two lengths). Thus, one performs a translation on the one interval to attempt to bring it into coincidence with the other. This empirical operation of equating intervals can be used as a basis for developing the properties of the interval scale, by postulating the existence of a group of suitable 'translations' such that one can define a binary (equivalence) relation $=D=$ between pairs of measure values. The relation $=D=$ holds for pairs $\left\langle m_{j}, m_{k}\right\rangle$ and $\left\langle m_{p}, m_{q}\right\rangle$ (i.e., the intervals between $\mathrm{m}_{j}$ and $\mathrm{m}_{\mathrm{k}}$ and between $\mathrm{m}_{\mathrm{p}}$ and $\mathrm{m}_{\mathrm{q}}$ are equal) if a translation in the group maps the endpoints $j$ and $k$ to endpoints $p$ and $q$, respectively. Thus, we might reasonably propose* the following postulate.

* I am indebted to Dr. Dirk vanDalen for several suggestions as to how to formalize this and other postulates in measurement theory. 
Postulate MP-4. - There exists a group (S,) of functions ("shifting functions") such that, for each $s \varepsilon S, s: M \rightarrow M$ is a monotonically increasing function, the group operation is composition of functions*, and $s\left(m_{i}\right)=m_{i} \rightarrow s=I$, where $I$ is the identity $\operatorname{map}\left(i . e ., s\left(m_{i}\right)=m_{i} \rightarrow s\left(m_{j}\right)=m_{j}\right)$ for all $\left.m_{j} \varepsilon M\right)$. We can define a relation $=\mathrm{D}=$ on the pairs $\left\langle\mathrm{m}_{j}, \mathrm{~m}_{\mathrm{k}}\right\rangle$ where $\mathrm{m}_{j}, \mathrm{~m}_{\mathrm{k}} \varepsilon \mathrm{M}$, such that, if $m_{j} \& m_{k}$ and $m_{p} \& m_{q}$, then $\left\langle m_{j}, m_{k}\right\rangle=D=\left\langle m_{p}, m_{q}\right\rangle$ if, and only if, for some $s \varepsilon s, m_{p}=s\left(m_{j}\right)$ and $m_{q}=s\left(m_{k}\right)$.

we shall sometimes write

$$
m_{j}-m_{k}=m_{p}-m_{q} \text { for }\left\langle m_{j}, m_{k}\right\rangle=D=\left\langle m_{p}, m_{q}\right\rangle,
$$

and may express either in words as "the interval (or distance) between $m_{j}$ and $m_{k}$ is equal to that between $m_{p}$ and $m_{q}$ "

It should be apparent that it is the form of the group (S, ) which will determine the nature of the "interval" of any particular generalized interval scale. (For example, we shall discuss a linear interval, for which $s=\{\mathrm{s} \mid \mathrm{s}=\mathrm{x}+\mathrm{b}\}$ and a logarithmic scale, for which $S=\{s \mid s=a x\}$, when $b$ and a are non-negative constants.)

The use of a group of monotonically increasing functions, which are also closely associated with invariance of ordinal scales, suggests this alternative postulate of the ability to equate 'intervals**.

Postulate MP-4'. - There exists a group $(S, \cdot)$ of monotonically increasing functions operating on $M$, such that, if $s \varepsilon S$, then

* That is, all the properties of a mathematical group are satisfied for $(S, \cdot)$. Thus, if $s \in S$, $t \varepsilon S$, then $t \cdot s \varepsilon S$, etc. (ref. 1 ). **The equivalence of Postulates MP-4 and MP-4' is only suggested here, and not proven. In fact, the formulation of interval scale postulates as presented here is quite tentative and open to further study. For example, it is not clear whether or not the generalized interval scale as developed here is of significant practical interest to measurement theory. It is, however, a concise generalization of interval concepts as they have been presented in stevens' work with linear and logarithmic interval scales. 
$s\left(m_{i}\right)=m_{i} \rightarrow s=I$, and such that we can define a relation $=D=$ on the pairs $\left\langle m_{j}, m_{k}\right\rangle(\varepsilon M \times M)$, with $\left\langle m_{j}, m_{k}\right\rangle=D=\left\langle m_{p}, m_{q}\right\rangle$ if, and only if, each interval

$$
\left\{m_{\ell} \mid m_{j} \leq m_{\ell}, m_{\ell} \leq m_{k} \text { and } m_{j}, m_{k}, m_{\ell} \varepsilon M\right\}
$$

taken as an ordinal scale is ordinally equivalent to the image interval

$$
\left\{m_{r} \mid m_{p} \leq m_{r^{\prime}} m_{r} \leq m_{q^{\prime}} \text { and } m_{p}=s\left(m_{j}\right), m_{q}=s\left(m_{k}\right)\right\}
$$

An immediate result of Postulate MP-4 (or MP-4') is the following theorem.

Theorem 6. - The relation $=\mathrm{D}=$ is an equivalence relation. Proof. - The relation $=D=$ is reflexive, since under the identity $\operatorname{map} m_{j}=I\left(m_{j}\right)$ and $m_{k}=I\left(m_{k}\right)$ and hence (since $I \varepsilon S$ for a group, and by definition of $=D=)\left\langle m_{j}, m_{k}\right\rangle=D=\left\langle m_{j}, m_{k}\right\rangle$. The relation is likewise symmetric, since by definition,

$$
\left\langle m_{j}, m_{k}\right\rangle=D=\left\langle m_{p}, m_{q}\right\rangle \rightarrow m_{p}=t\left(m_{j}\right), m_{q}=t\left(m_{k}\right),
$$

for some $t \varepsilon S$, and since an inverse map $t^{-1}$ exists in the group, for which $m_{j}=t^{-1}\left(m_{p}\right)$ and $m_{k}=t^{-1}\left(m_{q}\right)$, and thus

$$
\left\langle m_{p}, m_{q}\right\rangle=D=\left\langle m_{j}, m_{k}\right\rangle
$$

by definition. Transitivity of $=\mathrm{D}=$ also follows from the definition and group properties, as follows:

$$
\begin{aligned}
& \left\langle m_{j}, m_{k}\right\rangle=D=\left\langle m_{p}, m_{q}\right\rangle \text {, and } \\
& \left\langle m_{p}, m_{q}\right\rangle=D=\left\langle m_{r}, m_{s}\right\rangle \text {, together }
\end{aligned}
$$

imply all the following:

$$
\begin{aligned}
& t_{1}\left(m_{k}\right)=m_{p} ; t_{2}\left(m_{p}\right)=m_{r^{\prime}} \\
& t_{1}\left(m_{k}\right)=m_{q} ; t_{2}\left(m_{q}\right)=m_{s},
\end{aligned}
$$


for some $t_{1}, t_{2} \varepsilon S$. But by closure,

$$
\left(t_{1} \varepsilon S \text { and } t_{2} \varepsilon S\right) \rightarrow t_{2} \cdot t_{1} \varepsilon S
$$

Hence, $t_{2}\left[t_{1}\left(m_{j}\right)\right]=m_{r}$ and $t_{2}\left[t_{1}\left(m_{k}\right)\right]=m_{s}$ imply, by definition, that $\left\langle m_{j}, m_{k}\right\rangle=D=\left\langle m_{r}, m_{s}\right\rangle$.

We may now define a very general form of interval measurement as follows.

Definition 13

A generalized interval measurement is one satisfying Postulates MP-1, MP-2, MP-3, and MP-4 (or MP-4'). (Thus, a generalized interval measurement orders the measured set by $<f$, and permits establishing whether the 'interval' between any two measures is equal to that between any other two measures.) A generalized interval scale is the triple (D/ $\equiv_{f^{\prime}}\left\langle_{f} / \equiv_{f^{\prime}},(M \times M) /=D=\right)$ consisting of the equivalence classes of items measured, their relative ordering, and the equivalence classes of intervals between measures.

Clearly, each generalized interval measurement is an ordinal measurement. The measurements satisfying Postulate MP-4 are here called generalized interval measurements to distinguish them from stevens' "interval scales." This appears necessary because Postulate MP-4 as presented here appears to allow a more general interpretation of an 'interval' than Stevens' Zinear interval scale on the real numbers does. Postulate MP-4 suggests that any function which preserves order, matches endpoints, and is an element of a group of functions which do not allow "dilation" or change of interval whenever one point in the interval is held constant is a suitable 'translation' operation for interval comparison. This, in fact, allows a broader class of "intervalequating" operations than just the simple linear shifts achieved on number scales by subtraction. 
This point needs further discussion. Stevens' interval scale is in fact a linear interval scale, and may, for example, be contrasted with his recent presentation of a "logarithmic interval scale" (ref. 7, pp. 31-34). The linear interval scale can, for real-number measurements, establish equality of differences, such as

$$
7-2=9-4,
$$

whereas the logarithmic interval scale establishes equality of differences in logarithms of numbers (or, in other words, equality of ratios):

$$
\log a-\log b=\log c-\log a(\operatorname{or} a / b=c / d)
$$

Now, it happens that the present formalization of Postulate MP-4 is broad enough to include both these types of "intervals" (and many more). This may be an advantage, as a formal explication of the general concept of 'interval' (or it may, for other reasons, be a disadvantage). The best formalization of the postulate of interval equality is apparently a problem open to further study.

The formalization of interval equality as outlined in Postulate MP-4 does strongly suggest the central role played by the group $(S$,$) of shifting functions or "scale translations". For$ the linear interval scale on numbers, this group would be that subgroup of the group of monotonically increasing functions which corresponds to adding an arbitrary constant (number) to the given scale or subscale. Thus, we compare intervals on a linear scale by adding a constant number which will bring an endpoint of the interval into coincidence with the corresponding endpoint of the other interval. On the other hand, for the logarithmic interval

*A logarithmic scale is a generalized interval scale defined on the real numbers, for which the group $(S, r)$ is the group of functions of the form $S=m x$, where $m$ is a positive constant. 
scale, we add a logarithm of a number (or equivalently, multiply by a constant) to get two interval endpoints to coincide for comparison. Thus, for the logarithmic interval scale, the group $(S, \cdot)$ corresponds to the operations of multiplying measures by constants.

To restrict the group of shifting functions to those suitable for Stevens' interval scale requires adding considerable operational structure to the set $M$. "Subtraction" of elements is not defined on an arbitrary ordered set $M$. Although it is not certain that the required postulates would be entirely as extensive as those needed for the definition of integers (or any such subset of the reals) and for the definition of operations like addition and subtraction, it appears that very nearly the same amount of postulated structure and definitions of operations would be required, to suitably define linear intervals such as are used in Stevens' interval scales.

It should thus be clear why it is with the interval scales that one really begins to see the value of setting $M$ to be some subset of the real numbers. The real numbers exhibit all the necessary structure to provide for simple definition of linear intervals.

Until now, we have purposely avoided introducing the real numbers as the set $M$ of measures, because the intricate structure of the real numbers is not needed in developing such loosely structured sets as those used for nominal and ordinal measurements (or even generalized interval measurements). To bring in the reals at these stages in the hierarchy of scales could mislead one into conceiving that all the properties of the real numbers are available for postulating and discussing the properties of nominal, ordinal, and generalized interval scales. Besides, it would appear to be highly unsystematic to have introduced the numbers from the beginning and to have said (as Stevens' has essentially said) just that: with nominal scales we are neglecting all the properties of numbers except their equality 
or inequality; with ordinal scales we are considering only the identities and relative ordering of numbers; with interval scales we are considering only the identities, relative ordering, and interval equalities, etc. It is much more in keeping with mathematical methodology to postulate only the identities (and singlevalued functions) for nominal scales, to add on ordering as a further constraint to get the subclass of ordinal scales, then to add the assumption of interval equality to obtain interval scales, etc. Thus, instead of admitting a fully structured set and "wastefully" neglecting all its structure, we postulate the minimum needed to achieve each desired result.

It is only when we get close to having the full structure of the number system that is reasonable to introduce the numbers just to avoid the needless and tine-consuming repetition of many of the detailed postulates and properties of real numbers. Time does not permit the ideal detailed extension of the above procedure here, by the postulation of the minimum needed for each of the (linear) interval, ratio, and unique measurement scales we shall develop. Hence, after developing the concepts of scale equivalence and invariance for generalized interval scales, we shall compromise and introduce the real numbers to permit efficient discussion of the linear interval, ratio, and unique scales.

The shifting functions or translations discussed in connection with Postulate MP-4 and Definition 13 are operations that move one from part of a measuring scale to another part of the same scale, for generalized interval comparison. We may now consider associating two or more interval measurements in terms of equivalence.

Definition 14

Generalized interval measurements $f$ and $g$ are separationalzy equivalent if, and only if, for all $I_{j}, I_{k^{\prime}} I_{p^{\prime}} I_{q} \varepsilon D$,

$$
f\left(I_{j}\right)=f\left(I_{k}\right) \longleftrightarrow g\left(I_{j}\right)=g\left(I_{k}\right)
$$




$$
f\left(I_{j}\right)<f\left(I_{k}\right) \longleftrightarrow g\left(I_{j}\right)<g\left(I_{k}\right)
$$

and

$$
\begin{aligned}
& \left\langle f\left(I_{j}\right), f\left(I_{k}\right)\right\rangle=D=\left\langle f\left(I_{p}\right), f\left(I_{q}\right)\right\rangle \longleftrightarrow \\
& \left\langle g\left(I_{j}\right), g\left(I_{k}\right)\right\rangle=D=\left\langle g\left(I_{p}\right), g\left(I_{q}\right)\right\rangle .
\end{aligned}
$$

That is, two generalized interval measurements are separationally equivalent if, and only if, they are ordinally equivalent and establish the same set of equivalence classes of (generalized) intervals for elements of $D$.

Definition 15

A generalized interval scale is said to be separationally invariant under the set $T$ of transformations if, and only if, for all teT, $f$ and $t \cdot f$ are separationally equivalent. (Thus, $\left[D / \equiv_{f},{ }_{f} / \equiv_{f}, M /=D=\right]$ is separationally invariant under $T$ if, and only if, for all teT and all $I_{j}, I_{k^{\prime}} I_{p^{\prime}} I_{q} \varepsilon D$ :

$$
\begin{aligned}
& f\left(I_{j}\right)=f\left(I_{k}\right) \longleftrightarrow t\left[f\left(I_{j}\right)\right]=t\left[f\left(I_{k}\right)\right] \\
& f\left(I_{j}\right)<f\left(I_{k}\right) \longleftrightarrow t\left[f\left(I_{j}\right)\right]<t\left[f\left(I_{k}\right)\right]
\end{aligned}
$$

and

$$
\begin{aligned}
& f\left(I_{j}\right)-f\left(I_{k}\right)=D=f\left(I_{p}\right)-f\left(I_{q}\right) \longleftrightarrow \\
& \left.t\left[f\left(I_{j}\right)\right]-t\left[f\left(I_{k}\right)\right]=D=t\left[f\left(I_{p}\right)\right]-t\left[f\left(I_{q}\right)\right] .\right)
\end{aligned}
$$

Stevens has commented (ref. 1, p. 25) that a linear interval scale is invariant under the set of all linear transformations

$$
t\left[f\left(I_{j}\right)\right]=a f\left(I_{j}\right)+b
$$


where $\mathrm{a}$ and $\mathrm{b}$ are real constants. We, of course, can not claim such invariance properties for generalized interval scales since there are no defined addition and multiplication operations on the set $M$. However, it is clear that the generalized interval scale is at least separationally invariant under the set $s$ of monotonically increasing functions specified by Postulate MP-4: Theorem 7. - A generalized interval scale is separationally invariant under the set $S$ of transformations postulated in MP-4. Proof. - The proof follows immediately from Definition 15 and Postulate MP-4.

It is not difficult to find examples of separationally equivalent measurement scales, since one need merely pick two scales which are related by any shifting function in an appropriate group $(S, \cdot)$. For the special case of real number assignments, this might be, e.g., the group of functions $s(x)=x+a$, where a is some constant. Thus, for example, the Centigrade and Kelvin scales of temperature are separationally equivalent. But, a simple application of Definition 14 also shows that the Centigrade and Fahrenheit scales are also separationally equivalent. This is a case where real-number measurements related by a linear function $\mathrm{s}(\mathrm{x})=\mathrm{bx}+\mathrm{a}$ are separationally equivalent. Thus, we see that, although Theorem 7 is true, it is by no means the strongest theorem which might be presented about separational invariance.

\section{VII. - LINEAR INTERVAL SCALES}

In complexity measurements assigning real numbers, it has been found useful (ref. 6) to discuss the special case of separational equivalence of scales which are similar up to multiplicative and additive constants. To do so, one must define the special form of a linear interval scale, or as stevens' frequently refers to it, the interval scale. We begin by extending Postulate MP-4. 
Postulate $M P-4 R^{*}$. - The set $M$ is the set of real numbers, for which one can thus define equality of intervals as follows:

$$
\left\langle m_{j}, m_{k}\right\rangle=D=\left\langle m_{p}, m_{q}\right\rangle
$$

if, and only if, the real-number differences $m_{j}-m_{k}=m_{\ell}$ and $m_{p}-m_{q}=m_{n}$ are equal (i.e., $m_{\ell}=m_{n}$ ). Then we may state the obvious theorem:

Theorem 8. - For Postulate $M P-4 R,=D=$ is an equivalence relation. Proof. - The proof follows immediately from the definition of $=D=$ and the properties of number-theoretic differences.

It should be obvious that once Postulate MP-4R is assumed and the properties of real numbers are thus introduced, one can also naturally define an ordering $<_{D}$ of intervals, such that $\left\langle m_{j}, m_{k}\right\rangle{ }_{D}\left\langle m_{p}, m_{q}\right\rangle$ if, and only if, $m_{j}-m_{k}=m_{\ell}, m_{p}-m_{q}=m_{n}$, and $m_{\ell}<m_{n}$. Although in setting up empirical measurement scales using real numbers, there may not exist empirical operations corresponding to either interval equality or interval ordering (refs. $2,12,13,14$ ), in the mathematical sense one readily follows from the other once one assumes real-number measures. In accordance with stevens, we may now define an interval measurement.

Definition 16

An interval measurement (or, alternatively, a linear interval measurement) is one satisfying Postulates MP-1, MP-2, MP-3, and MP-4R. An interval scale (or, linear interval scale) is the triple (D/ $\equiv_{f}{ }_{f_{f}} / \equiv_{f}, R /=D=$ ) consisting of the equivalence classes of measured items, their relative ordering, and the equivalence classes of intervals between measures**.

*We use the notation $\mathrm{MP}-4 \mathrm{R}$ to mean measurement Postulate MP-4 for real-number assignments.

**Actually, the equivalence classes of intervals are simply a subset of the reals. 
Theorem 9. - An interval measurement is a generalized interval measurement.

Proof. - As mentioned in our discussion following Definition 13, the group $(S, \cdot)$ required for the linear interval scale to be a generalized interval measurement is the set of functions which add constant numbers to the measurements.

We may consider equating interval measurements by the following definition.

Definition 17

Interval measurements $f$ and $g$ are differentially equivalent if, and only if, for all $\mathrm{I}_{j}, \mathrm{I}_{\mathrm{k}}, \mathrm{I}_{\mathrm{p}}, \mathrm{I}_{\mathrm{q}} \in \mathrm{D}$,

$$
\begin{aligned}
& f\left(I_{j}\right)=f\left(I_{k}\right) \longleftrightarrow g\left(I_{j}\right)=g\left(I_{k}\right) \\
& f\left(I_{j}\right)<f\left(I_{k}\right) \longleftrightarrow g\left(I_{j}\right)<g\left(I_{k}\right)
\end{aligned}
$$

and

$$
\begin{aligned}
& f\left(I_{j}\right)-f\left(I_{k}\right)=f\left(I_{p}\right)-f\left(I_{q}\right) \longleftrightarrow \\
& g\left(I_{j}\right)-g\left(I_{k}\right)=g\left(I_{p}\right)-g\left(I_{q}\right) .
\end{aligned}
$$

Thus, two interval measurements are differentially equivalent if, and only if, they are separationally equivalent, with $=D=$ being equality of differences of real numbers.

Definition 18

An interval scale is said to be differentially invariant under the set $T$ of transformations if, and only if, for all tET, $f$ and $t \cdot f$ are differentially equivalent. (Thus, (D/ $\equiv_{f},<_{f} / \equiv_{f}$, $\mathrm{R} /=\mathrm{D}=$ ) is differentially invariant under $\mathrm{T}$ if, and only if, for all teT and all $I_{j}, I_{k^{\prime}}, I_{p}, I_{q} \varepsilon D$ :

$$
f\left(I_{j}\right)=f\left(I_{k}\right) \longleftrightarrow t\left[f\left(I_{j}\right)\right]=t\left[f\left(I_{k}\right)\right] \text {, }
$$




$$
f\left(I_{j}\right)<f\left(I_{k}\right) \longleftrightarrow t\left[f\left(I_{j}\right)\right]<t\left[f\left(I_{k}\right)\right]
$$

and

$$
\begin{aligned}
& f\left(I_{j}\right)-f\left(I_{k}\right)=f\left(I_{p}\right)-f\left(I_{q}\right) \longleftrightarrow \\
& t\left[f\left(I_{j}\right)\right]-t\left[f\left(I_{k}\right)\right]=t\left[f\left(I_{p}\right)\right]-t\left[f\left(I_{q}\right)\right] .
\end{aligned}
$$

Following Stevens, we may then state the following theorem. Theorem 10. - An interval scale is differentially invariant under the set of linear transformations $t(x)=a x+b$, for arbitrary constants $a$ and $b$.

Proof. -

$$
\begin{aligned}
& f\left(I_{j}\right)=f\left(I_{k}\right) \longleftrightarrow a f\left(I_{j}\right)+b=a f\left(I_{k}\right)+b \\
& f\left(I_{j}\right)<f\left(I_{k}\right) \longleftrightarrow a f\left(I_{j}\right)+b<a f\left(I_{k}\right)+b
\end{aligned}
$$

and

$$
\begin{aligned}
& f\left(I_{j}\right)-f\left(I_{k}\right)=f\left(I_{p}\right)-f\left(I_{q}\right) \longleftrightarrow \\
& a f\left(I_{j}\right)+b-\left[a f\left(I_{k}\right)+b\right]=a f\left(I_{p}\right)+b-\left[a f\left(I_{q}\right)+b\right] \cdot / / /
\end{aligned}
$$

Theorem 10 is, however, not the most general theorem possible concerning differential invariance. In parallel to our discussion following Theorem 7 , we can find cases of invariance which are not covered by Theorem 10. For example, the finite interval scales illustrated in Figure 4 are differentially equivalent but not related by a linear transformation of the form $t(x)=a x+b$. There exists, therefore, (at least for scales with finite numbers of elements) interval scales which are differentially equivalent but not related by a linear transformation $a x+b$. The case of infinite scales, and particularly those which are everywhere dense, may be of more interest, but we shall not here consider their relationship to the generality of Theorem 10. Suffice it 
to say that, in contrast to stevens' implications (ref. 1 , Table 6, ref. 2, Table 1) the linear transformations are not the only tramsformations under which an interval scale is invariant.

\begin{tabular}{|c|c|cc|}
$I_{j}$ & $f\left(I_{j}\right)=x_{j}$ & $g\left(I_{j}\right)=t\left[f\left(I_{j}\right)\right]$ \\
$a$ & 1 & 1 & \\
$b$ & 2 & 2 & $1 / 2$ \\
$c$ & 4 & 4 & $1 / 2$ \\
$d$ & 6 & 6 & $1 / 2$ \\
$e$ & 7 & 8 &
\end{tabular}

Figure 4. - Differentially equivalent scales which are not related by $a x+b$

\section{VIII. - RATIO AND UNIQUE SCALES}

We may now extend the hierarchy of measurement scales by recognizing those properties of the real numbers which lead to ratio scales*.

Postulate MP-5R. - One may establish equality of ratios, $\mathrm{m}_{\mathrm{j}} / \mathrm{m}_{\mathrm{k}}=r=\mathrm{m}_{\mathrm{p}} / \mathrm{m}_{\mathrm{q}}$, for measures in the set of real numbers, such that $m_{j} / m_{k} \stackrel{r}{=}=m_{p} / m_{q}$ if, and only if, for some $k \varepsilon R, m_{j}=k_{k}$ and $m_{p}=\mathrm{km}_{q}$ (or equivalently, $\log \mathrm{m}_{j}-\log \mathrm{m}_{\mathrm{k}}=\mathrm{D}=\log \mathrm{m}_{\mathrm{p}}-\log \mathrm{m}_{\mathrm{q}}$ ).

* A general postulate for ratio scales might be alternatively proposed in parallel fashion to that of Postulate MP-4, as follows. Postulate MP-5. - There exists a group (S,-) of functions such that, for each $s \varepsilon s, s: M \rightarrow M$ is a monotonically increasing function, the group operation - is composition of functions, and $s\left(m_{i}\right)=m_{i} \rightarrow m_{i}=0$, where 0 is thus a (unique) zero point. We define $=r=$ such that $\left\langle m_{j}, m_{k}\right\rangle=r=\left\langle m_{p}, m_{q}\right\rangle$ if, and only, $s\left(m_{k}\right)=m_{j}$ and $s\left(m_{q}\right)=m_{p}$, for some $s \in s$. The author is indebted to Dirk vanDalen, M.I.T., for suggesting this possible preliminary formulation of the postulates for a general ratio scale. The form of scale which satisfies MP-5 might, in parallel to Definition 13 , be called a "generalized ratio scale". 
Theorem 11. - The relation $=r=$ is an equivalence relation. Proof. The proof follows immediately from the definition of $=r=$ and the properties of division (or, subtraction) on the real numbers.

We shall be interested in preserving the $=r=$ equivalence of ratios like $\mathrm{m}_{j} / \mathrm{m}_{\mathrm{k}}$ and $\mathrm{m}_{\mathrm{p}} / \mathrm{m}_{\mathrm{q}}$, and thus will consider the equivalence classes $\mathrm{R} /=\mathrm{r}=$ as part of the structure of the ratio scale.

Definition 19

A ratio measurement is one satisfying Postulates MP-1, MP-2, MP-3, MP-4R, and MP-5R. A ratio scale is the ordered quadruple $\left\langle D / \equiv_{f},\left\langle{ }_{f} / \equiv_{f}, R /=D=, R /=r=\right\rangle\right.$ of equivalence classes of measured items, their relative ordering, the equivalence classes of linear intervals, and equivalence classes of ratios.

clearly, a ratio measurement is an interval measurement.

Although the properties of Postulate MP-5R follow directly from the known properties of real numbers, and thus follow directly from postulate MP-4R, we might conceive of an empirical measurement scale for which it were impossible to derive the one from the other, because of the lack of a known method for establishing a 'zero' on the empirical scale (ref. 2, pp. 31-34).

Without going into the details of the empirical counterparts of interval and ratio scales, we may merely note that the refinement which the ratio scale gives above that of an arbitrary interval scale is the ability to establish a unique, 'natural' zero point and, thus, to allow meaningful reference to ratios of measures (refs. 1,2,16). The Kelvin and Rankine scales of temperature are examples of such ratio scales.

We may note that it is conceivable that a scale may be developed which admits the equating of ratios such as postulated in MP-5R without admitting the equality of linear intervals as postulated in MP-4R. This is in fact what the logarithmic interval scale does, as indicated previously (Section VI), and as discussed in detail in reference 2, pp. 31-34. 
Equivalence and invariance of ratio scales may now be defined.

Definition 20

Ratio measurements $f$ and $g$ are proportionalzy equivalent if, and only if, for all $I_{j}, I_{k}, I_{p}, I_{q} \in D$,

$$
\begin{aligned}
& f\left(I_{j}\right)=f\left(I_{k}\right) \longleftrightarrow g\left(I_{j}\right)=g\left(I_{k}\right) \\
& f\left(I_{j}\right)<f\left(I_{k}\right) \longleftrightarrow g\left(I_{j}\right)<g\left(I_{k}\right) \\
& f\left(I_{j}\right)-f\left(I_{k}\right)=D=f\left(I_{p}\right)-f\left(I_{q}\right) \longleftrightarrow \\
& g\left(I_{j}\right)-g\left(I_{k}\right)=D=g\left(I_{p}\right)-g\left(I_{q}\right)
\end{aligned}
$$

and

$$
\begin{aligned}
& f\left(I_{j}\right) / f\left(I_{k}\right)=r=f\left(I_{p}\right) / f\left(I_{q}\right) \longleftrightarrow \\
& g\left(I_{j}\right) / g\left(I_{k}\right)=r=g\left(I_{p}\right) / g\left(I_{q}\right) .
\end{aligned}
$$

Thus, two ratio measurements are proportionally equivalent if, and only if, they are differentially equivalent and yield the same equivalence classes of ratios of measures.

Definition 21

A ratio scale is said to be proportionazly invariant under the set $T$ of transformations if, and only $i f$, for all teT, $f$ and $t \cdot f$ are proportionally equivalent.

Stevens has suggested the following theorem. Theorem 12. - A ratio scale is invariant under the set of "similarity transformations" $t(x)=$ ax; i.e., it is invariant under multiplication by any constant.

Proof. - The proof follows immediately from Definitions 20 and 21 and known properties of real numbers. 
Thus, multiplication by a constant preserves the form of a ratio scale. Scales like Rankine and Kelvin temperature scales are proportionally equivalent ratio scales related by a constant.

A quick check will show that the differentially equivalent scales of Figure 4 are not proportionally equivalent as ratio scales. Nor are scales with different zero points, such as Centigrade and Kelvin scales.

Proportional equivalence is a form of equivalence frequently useful in structural complexity measurements, where natural 'zeros' (no structure, etc.) may exist and scales may be "unique up to a constant multiplier."

To complete the hierarchy of scales we could now suggest the possibility of a natural unit of measurement, which restricted the 'constant multiplier' of a ratio scale to a single acceptable value, getting the following obvious development of a unique scale. Postulate MP-6R. - There is a unique unit of measurement. Definition 22

A unique measurement $f$ is one satisfying Postulates $M P-I$, $M P-2, M P-3, M P-4 R, M P-5 R$, and $M P-6 R$.

Definition 23

'Unique' measurements $f$ and $g$ are uniquely equivalent (or identical) if, and only if, $f\left(I_{j}\right)=g\left(I_{j}\right)$ for all $I_{j} \varepsilon D$.

Definition 24

A unique measurement is uniquely invariant under a set $T$ of transformations if, and only if, for all teT, $t \cdot f=f$. A unique scale is thus invariant under the identity transformation.

\section{IX. - THE 'SPECTRUM' OF MEASUREMENT SCALES}

We have a hierarchy or 'spectrum' of measurement scales, therefore, which may be usefully diagrammed as in Figure 5. This 


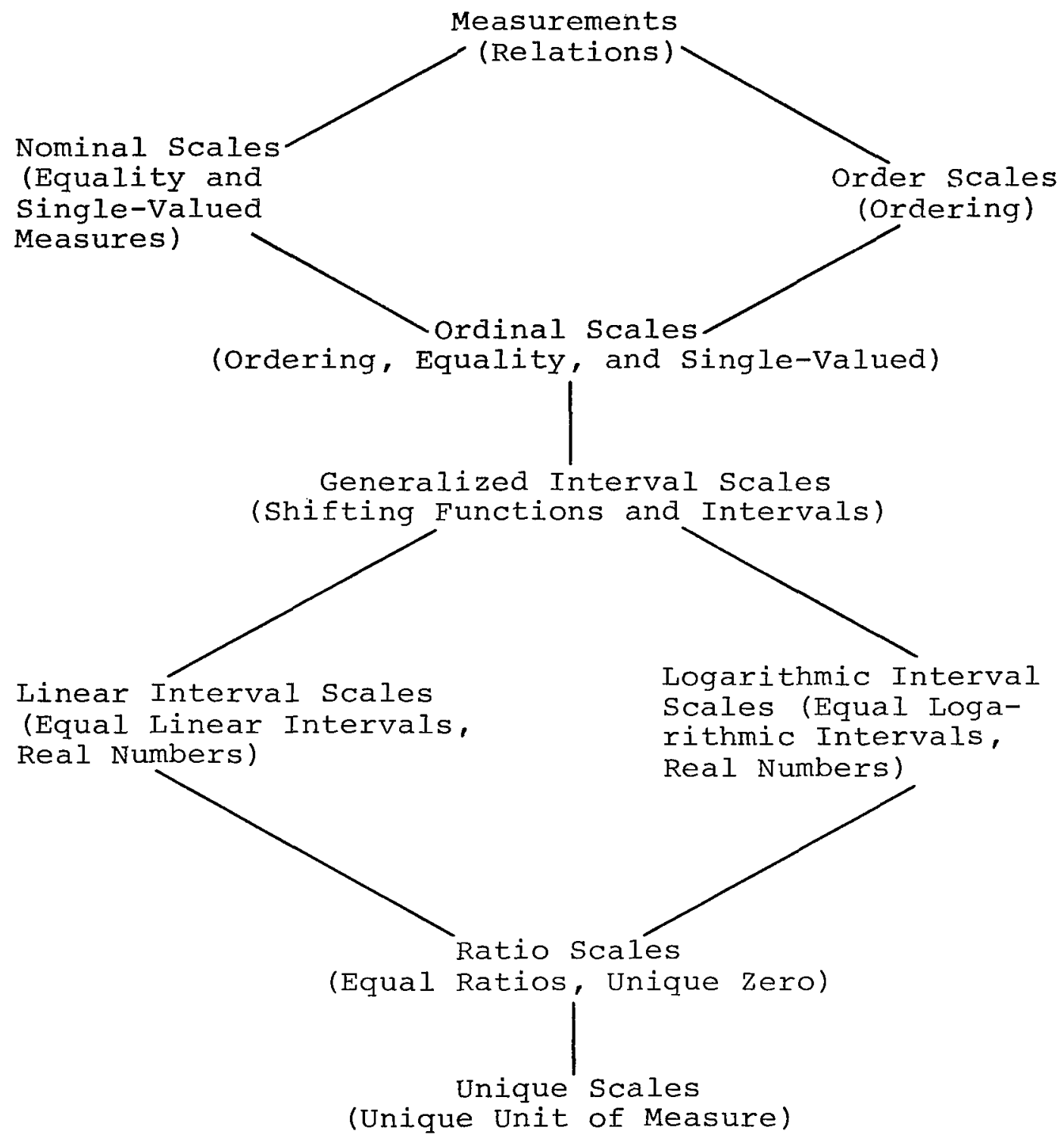

Figure 5. - The "spectrum" of measurement scale forms 
figure illustrates that nominal measurements are a subclass of all measurements, ordinal scales are a subset of the set of nominal scales, etc. (This diagramming is in accordance with a frequent practice of set-theoretic line diagrams (ref. 24, p. 11 or ref. 2, p. 32).) It is, no doubt, obvious that a number of other scale forms could be considered which may fall between the illustrated scale forms, or that may branch off from the spectrum at various points.

Figure 5 is a useful pictorial summary of the hierarchy of measurement scale forms and their associated restrictions upon the measurement functions and the structures of the sets of measure values. The definitions and theorems developed within the mathematical model presented in this paper illustrate the meaning and significance of equivalences, and invariances under sets of transformations for each of the scale types indicated in Figure 5. They also demonstrate to what degree stevens' claims about nominal, ordinal, interval, and ratio scales and their invariances are accurate.

It is appropriate, however, to note in passing, several interesting but unanswered questions about the hierarchy of measurement scales and their invariances. One such question concerns the nature of the maximal or largest set of transformations under which separational invariance of a generalized interval measurement is preserved. A similar question involves what maximal set of transformations preserve differential invariance of linear interval scales. The example of Figure 4 illustrates that this set must include more than just the linear transformations, contrary to stevens' implications (refs. 1,2). Another closely related question might then be what minimal additional condition must be added to those of differential equivalence to restrict the set of transformations (under which a linear internal scale is invariant) to being exactly the set of linear transforma- 
tions. This new form of restricted equivalence would then more closely correspond to stevens' implied scale invariance under only the linear transformations.

Electronics Research Center

National Aeronautics and Space Administration

Cambridge, Massachusetts, January 1969

$125-23-02-34-00$ 
REFERENCES

1. Stevens, S. S.: Mathematics, Measurement, and Psychophysics. Handbook of Experimental Psychology, Wiley, New York, 1951, pp. $1-49$.

2. Stevens, S. S.: Measurement, Psychophysics, and Utility. Measurement: Definitions and Theories. Wiley, New York, 1959, pp. 18-63.

3. Stevens, S. S.: The Psychophysics of Sensory Function. American Scientist, vol. 48, June 1960. Also in: Sensory Communication, M.I.T. Press, Cambridge, 1961, pp. 1-34.

4. Adams, E. W., Fagot, R. F., and Robinson, R. E.: A Theory of Appropriate Statistics. Psychometrica, vol. 30, 1965, pp. $99-127$.

5. Grunstra, B.: On Distinguishing Types of Measurement. Boston Studies in the Philosophy of Science, vol. 5, Boston, 1967.

6. Lea, W. A.: Measurement of Syntactical Complexity of Formal Expressions. Dissertation for the Degrees of Master of Science and Electrical Engineer, Department of Electrical Engineering, Massachusetts Institute of Technology, Cambridge, 1966 .

7. Stevens, S. S.: Measurement, Statistics, and the Schemapiric View. Science, vol. 161, no. 3844, 30 August 1968, pp. 849-856.

8. Suppes, P., and Zinnes, J. I.: Basic Measurement Theory. Handbook of Mathematical Psychology, vol. 1, Wiley, New York, 1963 , pp. $1-76$.

9. Campbel1, N. R.: An Account of the Principles of Measurement and Calculation. Longmans, Green, London, 1928.

10. Campbe11, N. R.: Symposium: Measurement and Its Importance for Philosophy. Aristotelian Society Supplement, vol. 17, Harrison, London, 1938.

11. Caws, P.: Definition and Measurement in Physics - Measurement: Definitions and Theories. Wiley, New York, 1959, pp. 3-17.

12. Coombs, C. H.: Psychological scaling without a Unit of Measurement. Psychological Review, vol. 57, 1950, pp. 145-158.

13. Coombs, C. H.: A Theory of Scaling. University of Michigan Press, Ann Arbor, 1952. 
14. Coombs, C. H., Raiffa, H., and Thrall, R. M.: Some Views on Mathematical Models and Measurement Theory. Psychological Review, vol. 61, 1954, pp. 132-144.

15. Suppes, P.: Measurements, Empirical Meaningfulness, and Three-Valued Logic-Measurement: Definitions and Theories. Wiley, New York, 1959, pp. 129-143.

16. Von Neumann, J., and Morganstern, O.: Theory of Games and Economic Behavior, ch. 3, Wiley (Science Editions), New York, 1964.

17. Kleene, S. C.: Introduction to Meta-Mathematics. Van Nostrand, Princeton, 1952 .

18. Mendelson, E.: Introduction to Mathematical Logic. Van Nostrand, 1964 .

19. Bruner, J. S., Goodman, J. S., and Austin, G. A.: A Study of Thinking. Wiley, New York, 1956.

20. Ginsburg, S.: An Introduction to Mathematical Machine Theory. Addison Wesley, Reading, Mass., 1962.

21. Russell, B.: Introduction to Mathematical Philosophy . MacMillan, New York, 1920.

22. Caldwel1, S. H.: Switching Circuits and Logical Design . Wiley, New York, 1958 . 


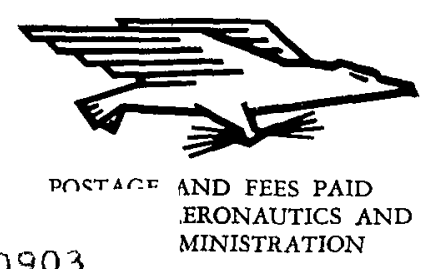

O4U OO1 $445130 S \quad 6916300903$
AIR FORCE WEAPONS LABCRATORY/AFWL/
KIRTLAND AIR FURCE BASE. NEW MEXICO $8711 \%$

ATR E LUU BOHMAN ACTINC CHIEF TECH. LIT

POSTMASTER: If Undeliverable (Section 158

"The aeronantical and space activities of the United States shall be conducted so as to contribute. . . to the expansion of buman knowledge of phenionena in the atmosphere and space. The Administration sball provide for the usidest practicable and appropriate dissemination of information concerning its activities and the results thereof."

- National Aeronautics and Space Act of 1958

\title{
NASA SCIENTIFIC AND TECHNICAL PUBLICATIONS
}

TECHNICAL REPORTS: Scientific and technical information considered important, complete, and a lasting contribution to existing knowledge.

TECHNICAL NOTES: Information less broad in scope but nevertheless of importance as a contribution to existing knowledge.

TECHNICAL MEMORANDUMS:

Information receiving limited distribution because of preliminary data, security classification, or other reasons.

CONTRACTOR REPORTS: Scientific and technical information generated under a NASA contract or grant and considered an important contribution to existing knowledge.
TECHNICAL TRANSLATIONS: Information published in a foreign language considered to merit NASA distribution in English.

SPECIAL PUBLICATIONS: Information derived from or of value to NASA activities. Publications include conference proceedings, monographs, data compilations, handbooks, sourcebooks, and special bibliographies.

\section{TECHNOLOGY UTILIZATION}

PUBLICATIONS: Information on technology used by NASA that may be of particular interest in commercial and other non-aerospace applications. Publications include Tech Briefs, Technology Utilization Reports and Notes, and Technology Surveys.

Details on the availability of these publications may be obtained from:

\author{
SCIENTIFIC AND TECHNICAL INFORMATION DIVISION \\ NATIONAL AERONAUTICS AND SPACE ADMINISTRATION \\ Washington, D.C. 20546
}

